Ann. Sci. forest., 1971, 28 (1), 5-22.

\title{
PREMIÈRES RECHERCHES \\ SUR LE DÉTERMINISME DE L'ACROTONIE DES VÉGÉTAUX LIGNEUX
}

\author{
P. CHAMPAGNAT, P. BARNOLA et Suzanne LAVARENNE \\ avec la collaboration technique de Françoise Aulagner et Simone GenestıER \\ que nous remercions très vivement
}

Laboratoire de Phytomorphogénèse, associé au C.N.R.S., 4, rue Ledru, 63 - Clermont-Ferrand

\section{RÉSUMÉ}

Sous le climat du centre de la France, la préséance des bourgeons apicaux qui conditionne l'acrotonie des 4 espèces d'arbres étudiées n'est pas en place avant le mois de mars. Elle fait intervenir des corrélations actives à un moment où aucune croissance n'est décelable : le comportement comparé des boutures de nœuds isolés et des rameaux entiers prouve qu'à une basitonie automnale identique à celle des buissons (Rubus, Sambucus, etc.), succède une aptitude au développement comparable pour tous les bourgeons, puis une préséance apicale beaucoup moins intense sur le nœud isolé que sur le rameau entier. L'intégrité de ce rameau est ainsi un facteur important de l'acrotonie, que le système radiculaire peut aussi renforcer.

L'acrotonie n'est donc pas directement comparable à la basitonie : elle lui succède et se substitue à elle. Elle met en jeu des facteurs originaux, de nature encore inconnue, qui précisément empêchent la tendance basitone de s'exprimer.

Ces résultats confirment en outre l'existence constante de dormances d'intensité variée le long du rameau et une modification progressive, d'octobre à mars, du gradient d'aptitude à la croissance ainsi créé.

Les travaux de Troll (1939) et Rauh (1939) ont montré que l'acrotonie conditionne le port des arbres alors que la basitonie est le principe essentiel du buissonnement. Sur un rameau acrotone, seuls les bourgeons apicaux engendrent des pousses longues, les autres donnent des rameaux courts ou restent latents. Parfois la différenciation apparaît dès le débourrement (acrotonie de débourrement); parfois la plupart des bourgeons commencent à se développer uniformément avant que les apicaux poursuivent seuls leur croissance (acrotonie par croissance différentielle) (CHAMPagnat, 1965). 
Si on supprime l'extrémité du rameau, avant ou après le débourrement, on modifie, en général, le devenir de certains yeux : les plus proches du point de taille, qui, sans cela, auraient peu évolué, donnent des pousses longues. Ils étaient donc soumis à une inhibition corrélative de la part de ceux qui ont été supprimés.

On parle souvent dans ce cas d'une dominance apicale. Il ne s'agit pourtant pas de l'influence exercée par un point végétatif principal sur les ébauches axillaires qu'il a engendrées. Si l'espèce est sympodiale, il s'agit de corrélations entre bourgeons frères; si elle est monopodiale, et si la tige est assez vigoureuse, on est en présence de l'action conjuguée du bourgeon terminal et des axillaires sous-jacents sur ceux qui sont insérés plus bas. On est, en tout cas, en présence de l'action d'un ensemble assez mal défini de méristèmes, sur un autre groupe. Les auteurs anglo-saxons qui admettent cette distinction opposent maintenant «apical control à «apical dominance » (PHILIPPS, 1969).

Nous préférons, pour notre part, parler d'un gradient de préséances, établi ou fixé le long du rameau (Champagnat, 1964, 1965) qui préexiste aux inhibitions corrélatives et les rend possibles et insister sur le fait que ce gradient n'existait pas sur la pousse herbacée, siège d'une dominance apicale typique, mais qu'il s'est établi après la fin de sa croissance en longueur et avant le réveil de la végétation au printemps suivant, donc pendant l'automne et l'hiver, saisons au cours desquelles évolue la dormance des bourgeons.

Cette dormance est définie classiquement comme un caractère de chaque bourgeon, comme une inaptitude totale ou partielle qu'il a à reprendre sans délai une croissance rapide lorsqu'il est placé dans les conditions de milieu les plus favorables. Elle ne suppose aucune corrélation entre points végétatifs.

Il est évident que si l'évolution de la dormance n'est pas uniforme sur toute la longueur d'une tige, certains bourgeons libérés plus tôt deviendront plus aptes que d'autres à répondre aux sollicitations d'un milieu favorable. Ils évolueront plus vite ou plus tôt et pourront ensuite inhiber corrélativement les autres, encore partiellement endormis. Une sortie plus rapide de dormance serait dans ce cas le facteur de préséances (BARNóa, 1969, Champagnat, 1969) responsable du mode de ramification du rameau au printemps.

Si ce sont les bourgeons les plus proches du sommet qui possèdent cette préséance on observera une acrotonie; si ce sont les plus voisins de la base c'est une basitonie qui s'installera.

La valeur de ce raisonnement peut être soumise à l'analyse expérimentale.

On peut étudier, de septembre à mars, l'aptitude à la croissance de bourgeons isolés, prélevés, avec une partie des entre-nœuds adjacents, à différents niveaux sur le rameau, et placés dans des conditions de milieu favorables. On travaillera notamment dans une gamme étendue de températures puisqu'il est prouvé que l'évolution d'une dormance se traduit par une modification de l'éventail des températures où une croissance est possible (VÉGIS, 1961, 1965).

C'est en utilisant une telle technique que Barnola a pu démontrer que la basitonie d'un Rubus $(1969,1970)$ ou d'un Sureau (1971) provient, de ce qu'en automne, les bourgeons de la base, moins dormants, sont capables de croître à des températures basses $\left(8^{\circ}, 12^{\circ}, 15^{\circ}\right)$ alors que ceux du reste de la tige en sont incapables. A la fin de l'hiver les premiers sont devenus très gros et ont acquis une préséance définitive. 
On pourrait donc, par analogie, penser que, sur une espèce acrotone, les bourgeons les plus apicaux sont les moins dormants et acquièrent ainsi leur possibilité de dominance.

Quelques faits connus donnent à penser que la réalité est plus complexe :

a) CrABbÉ a prouvé qu'en septembre et octobre, c'étaient les bourgeons de la base d'un rameau de Pommier ou de Poirier qui, comme ceux d'un Rubus ou d'un Sureau, possédaient la meilleure aptitude au développement. Ce qui est original, c'est que cette aptitude disparaît au cours de l'hiver et est remplacée par une assez nette préséance des méristèmes apicaux (1968).

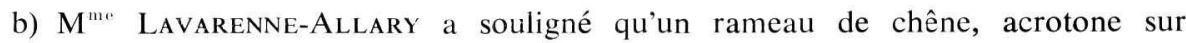
l'arbre, dans la nature, ne l'est pas s'il est isolé et mis à débourrer à la température du laboratoire, en février ou mars. Il peut le devenir après une injection apicale de certaines substances de croissance et notamment de cytokinines (1965).

c) Sur la Vigne, Huglin a montré que ces méristèmes apicaux étaient, dès le début de l'automne, les moins dormants: de jeunes plantes rentrées en serre avant les froids sont, en effet, nettement acrotones alors que, si elles sont soumises plus tard dans la saison aux conditions de milieu favorable, elles se ramifient sur toute leur longueur ou même sont légèrement basitones (1965; BUGNON et BESsis, 1968).

d) On peut enfin rappeler que, sur le Framboisier, si on excepte les bourgeons de la base dont la préséance vient d'être soulignée, un rameau entier prélevé dans la nature et placé à températures élevées au début de l'automne est acrotone comme un sarment de vigne à la même époque. Comme lui, il devient le siège d'un débourrement généralisé lorsqu'il a subi le froid hivernal pendant quelques semaines (BARNOLA, 1969).

e) Au contraire, si aux mêmes dates, on étudie le pouvoir de croissance de bourgeons isolés sur ces mêmes pousses de Rubus, on constate qu'en septembre ou octobre, les méristèmes de la région apicale sont ceux qui possèdent les plus faibles potentialités (BARNola, 1969 ; BeN AMOR, 1970). L'acrotonie demande donc pour s'exprimer précocement l'intégrité de la tige, ce qui laisse supposer l'intervention de corrélations de nature inconnue, à une époque où il est classique d'admettre qu'il n'en existe pas.

C'est donc dans le but d'aboutir à une meilleure connaissance de ces déterminismes que nous avons entrepris les recherches dont les premiers résultats sont résumés dans ce mémoire.

\section{MATÉRIEL ET MÉTHODES}

Nous avons voulu :

1) Comparer un nombre assez grand d'espèces possédant des caractères morphologiques différents: le frêne (Fraxinus excelsior L.) présente dans la nature, sur des rameaux vigoureux, une acrotonie par croissance différentielle alors que sur de jeunes plantes issues de semis on observe une acrotonie de débourrement. Il s'agit en outre d'un arbre typique, monopodial, pris comme modèle par TroLl. Le Tilleul (Tilia platyphyllos Scop.) est également un arbre typique mais sympodial et dont l'acrotonie par croissance différentielle est particulièrement caractéristique. Le Cerisier (Prunus avium L.) peut permettre d'utiles comparaisons avec les arbres fruitiers à pépins (sur lesquels travaille CrABBÉ); il possède en outre, très souvent, un groupe de bourgeons apicaux à préséance marquée, responsable d'une ramification en étages superposés, comparable, bien que moins typique, à celle d'un Rhamnus frangula L. ou de certaines espèces tropicales (Halle et Oldman, 1970 ; PrÉvost, 1966). Le 
Charme (Carpinus betulus L.) enfin possède comme le Tilleul une acrotonie par croissance différentielle, mais il exprime souvent une aptitude au buissonnement qui le rend assez proche de certaines espèces basitones plus spécialement étudiées par l'un de nous (BARNOLA, 1969).

2) Analyser, séparément, d'octobre à mars, dans une gamme de températures allant de $8^{\circ}$ à $27^{\circ}$, le comportement de bourgeons isolés sur des boutures de nouds et celui de rameaux isolés entiers non ébourgeonnés pour mieux préciser ce qui, dans l'évolution des tendances morphogénétiques, revient aux propriétés intrinsèques (fix ces) de chaque bourgeon et aux curieuses corrélations signalées ci-dessus. De jeunes plantes pourvues ou non de leur système radiculaire ont parfois été utilisées pour faciliter la comparaison.

En général, des pousses de l'année, prélevées sur des arbres âgés de deux ou trois ans, sont récoltées dans la nature de préférence dans une même station et sont choisies de même vigueur moyenne.

Les rameaux d'un an sont répartis en deux séries:

$1^{\circ}$ dans la première, ils sont placés, intacts, horizontalement, dans des gouttières en matière plastique de $2 \mathrm{~m}$ de long et recouverts de tourbe ;

$2^{\circ}$ dans la deuxième, les rameaux sont découpés, en boutures de nœuds isolés pris à trois niveaux différents: sommet, milieu, base. Ces boutures sont placées verticalement dans des boîtes en matière plastique remplies de sable humide et percées de trous à la base pour éviter un excès d'humidité. Pour empêcher toute dessiccation de l'échantillon, les sections supérieures des boutures ont été au préalable paraffinées.

Toutes ces expériences sont réalisées dans des pièces obscures dont l'humidité varie entre 60 et $70 \%$ et à des températures allant, comme nous l'avons déjà mentionné, de $8^{\circ} \mathrm{C} \pm 1^{\circ} \mathrm{C}$ à $30^{\circ} \mathrm{C} \pm 1^{\circ} \mathrm{C}$ (voir graphique). Leur durée est, en moyenne, de 6 semaines.

Chaque gouttière renferme 20 rameaux entiers, chaque boîte en plastique contient une série de 20 boutures de nœuds isolés correspondant à un des 3 niveaux définis précédemment.

Dans chaque condition de température, on compare l'évolution de ces bourgeons. Une fois par semaine, les résultats sont relevés de la façon suivante:

\section{a. - Boutures de nouds isolés}

Pour chaque niveau, on note le nombre de bourgeons débourrés par rapport au nombre total de bourgeons. On considère qu'un bourgeon est débourré lorsque les écailles s'écartant, l'ébauche de la première feuille apparait.

\section{b. - Rameaux entiers}

La même méthode que précédemment est employée. Il faut cependant préciser que pour permettre une comparaison avec les boutures de nouds isolés, nous observons les bourgeons à des niveaux identiques, c'est-à-dire : 2 bourgeons de la partie apicale, 2 de la partie médiane, 2 de la partie basale.

Des essais préliminaires nous ont permis d'étudier l'influence, qui est négligeable, de la longueur du mérithalle, de la position des boutures et des rameaux entiers, et du milieu de culture (sable ou tourbe).

Pour simplifier et par souci d'unité, les seuls résultats présentés sous forme d'histogrammes sont ceux obtenus 40 à 50 jours après le commencement de l'expérience, donc peu avant la fin de celle-ci. Ils sont toujours de même sens que ceux relevés les semaines précédentes, mais ne sont pas obligatoirement ceux qui montrent le mieux la différence de comportement des bourgeons des différents niveaux. Le calcul du temps harmonique moyen de débourrement (voir fig. 6) n’apporte aucun élément nouveau, susceptible de modifier nos conclusions.

\section{RÉSULTATS OBTENUS ET DISCUSSION}

$$
\text { A. - Cas du Tilleul }
$$

a) Boutures de nouds isolés (fig. 1 à 6).

$1^{\circ}$ D'octobre à décembre on observe un développement presque uniquement basitone ; plus précisément, en octobre, au bout de 40 à 50 jours les débourrements inté- 
Boutures de THLEIJL

OCTOBRE

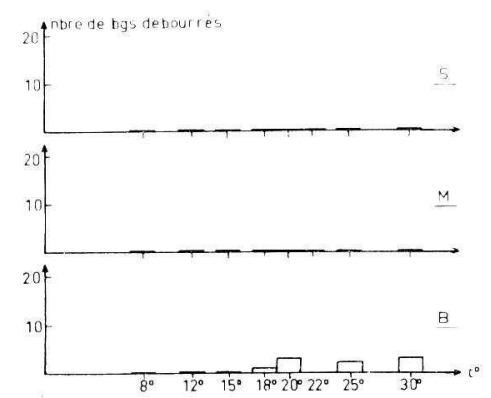

FIG. 1

Boutures de TILLEUL DECEMBRE

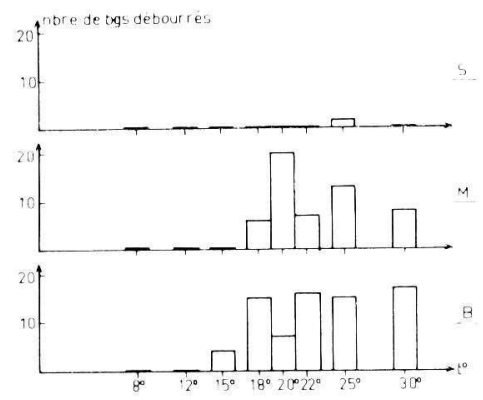

FIG. 3

Boutures de TILLEUL FEVRIER

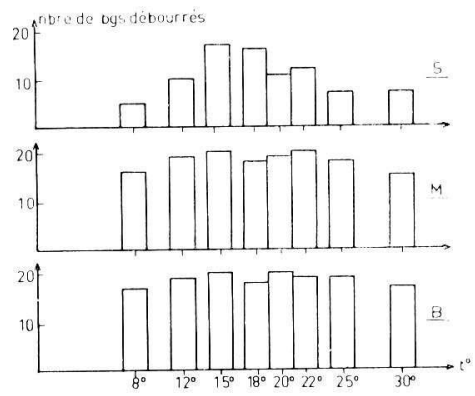

FIG. 5

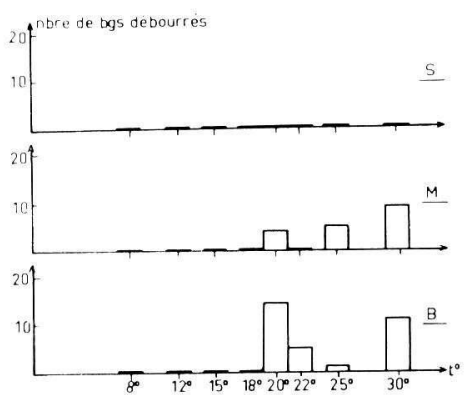

FIG. 2

Boutures de TI LLEUI IANVIER

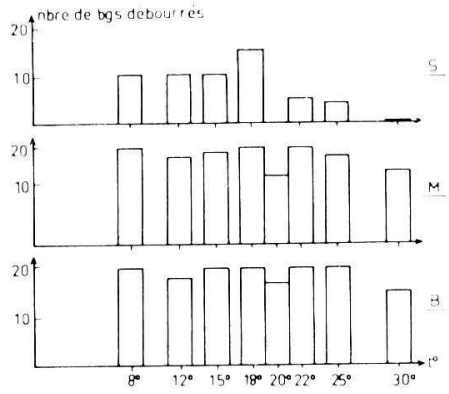

FIG. 4

Boutures de TILLEUL MARS

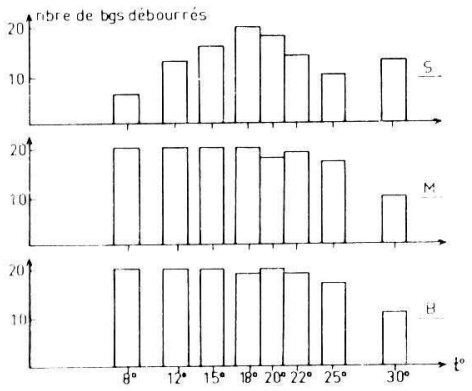

FIG. 6

FIG. 1 à 6. - Débourrement des bourgeons sur des boutures de nouds isolés de Tilia platyphylios prélevées dans la nature entre octobre et mars et maintenues à différentes températures (abscisses)

Chaque série comprend 20 boutures (ordonnées). Les résultats sont relevés 40 à 50 jours après la mise en expérience.

S; M; B : boutures prélevées respectivement dans les régions apicale, médiane et basale aes rameaux. 
ressent uniquement les bourgeons de la base des rameaux; ils restent rares et sont conditionnés par des températures élevées (supérieures à 20 $0^{\circ}$. Ce caractère se maintient en novembre, mais 50 à $70 \%$ des boutures sont devenus capables de croître. En même temps quelques yeux de la partie médiane évoluent. En décembre, à partir de $18^{\circ}$, donc sans grande ouverture de l'éventail des températures, ce débourrement se généralise aux 2/3 inférieurs des tiges alors que les méristèmes apicaux restent latents pendant toute la durée de l'expérience.

On est donc en présence d'une basitonie potentielle nette, presque aussi bien caractérisée que celle d'un Rubus et plus durable que celle d'un Sambucus (BARNola, 1971). Ce qui est nouveau, c'est qu'elle ne s'exprime, dans la nature, par aucune croissance différentielle des bourgeons de base, donc qu'elle ne se traduit par aucune prise de préséances.

$2^{\circ}$ Entre décembre et janvier, les processus qui déplaçaient vers l'apex le pouvoir de croissance des bourgeons s'accélèrent considérablement.

Non seulement tous ceux de la partie basale et médiane évoluent rapidement (les temps de latence pour que $50 \%$ d'entre eux débourrent sont voisins de 20 jours à $20^{\circ}$ alors qu'ils atteignaient 40 jours en novembre et 30 jours en décembre), mais encore une partie des apicaux s'épanouissent (temps de latence moyen voisin de 40 jours à $20^{\circ}$ ). De plus, l'éventail des températures efficaces s'est ouvert au maximum puisqu'à $8^{\circ}$, les résultats sont aussi bons qu'à $18^{\circ}$ ou $20^{\circ}$. En février et mars aucun fait vraiment nouveau n'est à signaler: les potentialités des bourgeons, tout le long de l'axe qui les porte, sont voisines. Tout au plus peut-on noter que la température de $30^{\circ}$ est la moins favorable et qu'entre $8^{\circ}$ et $15^{\circ}$ les boutures apicales restent assez nettement défavorisées par rapport aux autres.

$3^{\circ}$ En aucun cas, donc, nous n'assistons à une prise de préséance des éléments apicaux comparables à celle notée par CrabBé sur le Pommier et le Poirier (1968).

Tout au plus observe-t-on une égalisation des chances de développement de chaque point végétatif. Le fait n'est, d'ailleurs, pas étonnant. Des rameaux vigoureux de Tilleul, tels que ceux utilisés dans ces expériences ont, dans la nature, une acrotonie par croissance différentielle: la plupart des bourgeons débourrent en même temps et c'est plus tard que, par leur allongement plus rapide et plus durable, les éléments apicaux manifestent leur préséance. Une égalisation des chances, à la fin de l'hiver pourrait donc paraitre suffisante pour expliquer ce comportement. On doit, cependant, noter que, toujours dans la nature, quelques bourgeons basaux restent latents. Ils ont pourtant, lorsqu'on les isole, des aptitudes à croître au moins aussi bonnes que celles des autres. S'ils ne les expriment pas c'est, sans doute que, sur le rameau entier, ils sont soumis à des corrélations d'inhibition avant qu'une évolution morphologique soit décelable.

b) Rameaux entiers (fig. 7 à 9).

Ils manifestent tardivement, mais clairement, l'existence de ces corrélations:

$1^{\circ}$ En octobre ils ne se distinguent pas des boutures de nouds isolés; le petit nombre de bourgeons qui débourre est l'indice d'une basitonie qui ne se manifeste qu'aux températures élevées. 


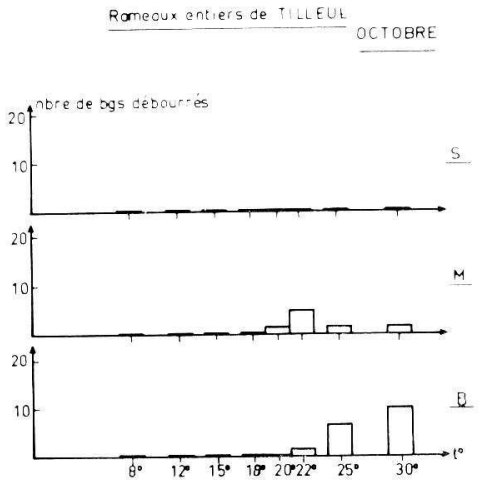

FIG. 7
Romeaux entiers de TILLEUL

JANV|ER

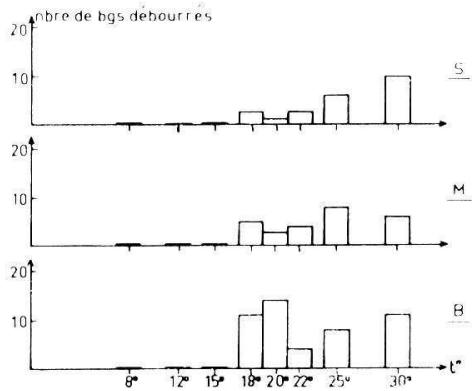

FIG. 8

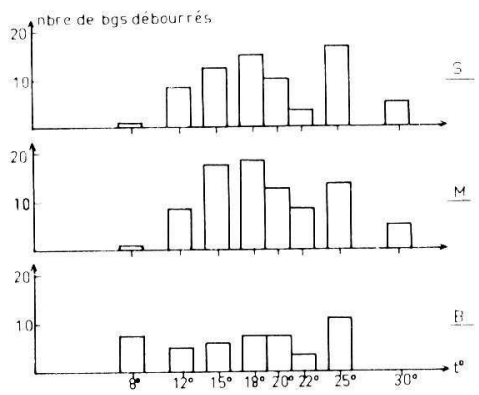

FIG. 9

FIG. 7 à 9. - Débourrement des bourgeons sur des rameaux entiers de Tilia platyphyllos prélevés dans la nature entre octobre et mars et maintenus à différentes températures (abscisses)

Chaque série comprend 20 boutures (ordonnées). Les résultats sont relevés 40 à 50 jours après la mise en expérience. Ils découlent de l'observation de 3 paires de bourgeons de chaque pousse: une paire apicale (S), une paire médiane $(\mathrm{M})$ et une paire basale (B), ce qui facilite la comparaison avec les boutures de nœuds isolés (fig. 1 à 6 ). 
$2^{\circ}$ En janvier le gradient n'est pas renversé. La tendance basitone est même plus nette que sur les boutures, mais $50 \%$ au plus des bourgeons débourrent, alors que la croissance était générale après leur isolement.

$3^{\circ}$ C'est en mars qu'on observe un fait nouveau. La plupart des bourgeons médians et apicaux croissent à peu près simultanément entre $15^{\circ}$ et $25^{\circ}$. Ce sont ceux de la base qui reprennent leur activité le plus tard et le plus irrégulièrement $(50 \%$ environ restent latents à toutes les températures). On retrouve donc le comportement observé dans la nature: le gradient favorable à l'expression de l'acrotonie par croissance différentielle est en place.

$4^{\circ}$ Il faut pourtant remarquer qu'à $8^{\circ}$ le débourrement est plus rapide à la base. De nouveaux essais sont cependant nécessaires pour expliquer ce fait. Il pourrait signifier que les préséances résultant de l'intégrité du rameau ont besoin de températures assez élevées pour se manifester.

On peut donc retenir que :

a) L'isolement des bourgeons supprime les corrélations responsables de la ramification normale, corrélations qui sont assez fortes pour empêcher l'aptitude à la croissance de certains points végétatifs de se manifester. Deux gradients de préséances entrent donc en conflit à une époque où l'activité physiologique des tiges est faible. Les tendances morphogènes ne résultent pas seulement d'une évolution différente de la dormance de chaque bourgeon, considéré comme une unité indépendante, le long de l'axe.

b) La mise en conditions favorables de rameaux entiers conduit souvent à un débourrement plus lent et moins général que celle des nœuds isolés. Le fait est surtout net en décembre et janvier. Il nous est pour l'instant impossible d'en donner une explication, mais nous montrerons plus loin qu'il se retrouve sur les autres espèces.

\section{B. - Cas du Frêne}

a) Boutures de nueuds isolés prélevées sur des rameaux vigoureux (fig. 10 à 13).

Les résultats sont comparables à ceux obtenus avec le Tilleul. Quelques différences d'importance secondaire peuvent cependant être signalées.

$1^{\circ}$ La basitonie est bien marquée d'octobre à décembre, surtout à $20^{\circ}$ et $22^{\circ}$, malgré quelques débourrements apicaux qui n'existaient pas sur le Tilleul. Aucun développement n'est décelable après 2 mois, aux températures basses. A $25^{\circ}$ et $30^{\circ}$ la réaction est également moins intense.

$2^{\circ}$ En janvier, cet éventail s'est légèrement ouvert, mais l'intensité des dormances est plus forte que sur le Tilleul à pareille époque. Le gradient médiobasitone reste net dans les deux cas.

$3^{\circ}$ En mars, on note la principale différence entre les deux espèces : ce gradient n'évolue pas. Aucune tendance à un débourrement généralisé ou à une acrotonie ne se manifeste. Le comportement des boutures ne rend donc pas compte du mode de ramification observé dans la nature qui, rappelons-le, commence par une croissance simultanée de la plupart des bourgeons (à l'exception des plus proches de la 
Boutures de FRÊNE OCTOBRE

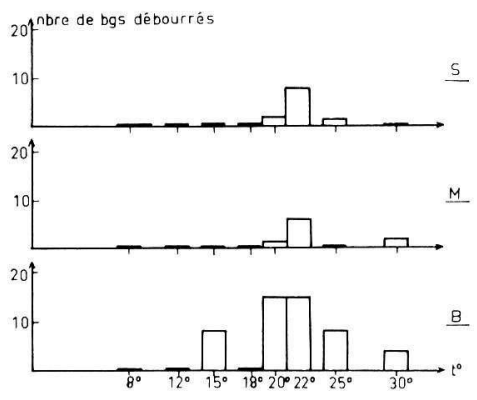

FIG. 10
Ecutures ce =REेV

NOVEMBPE

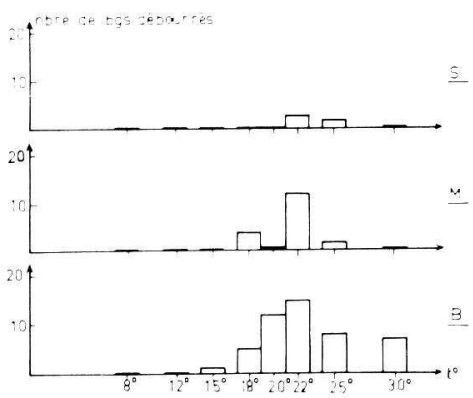

FIG. 11
Boutures de FRENE

JANVIER

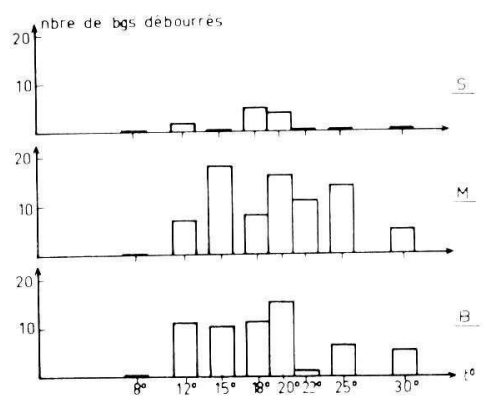

FIG. 12
Boutures de FRENE

MARS

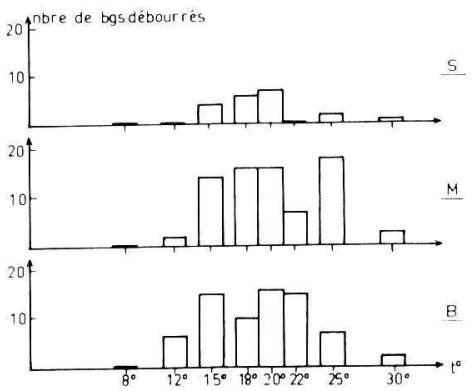

FIG. 13

FIG. 10 à 13. - Débourrement des bourgeons sur des boutures de nauds isolés de Fraxinus excelsior prélevées dans la nature entre octobre et mars et maintenues à différentes températures (abscisses)

Chaque série comprend 20 boutures (ordonnées). Les résultats sont relevés 40 à 50 jours après la mise en expérience.

S; $\mathbf{M} ; \mathrm{B}$ : boutures prélevées respectivement dans les régions apicale, médiane et basale des rameaux. 
base) suivie par une prise de préséance apicale. Il n'est, cependant, pas impossible que l'évolution observée dès février sur le tilleul se produise brusquement en avril, peu de jours avant la reprise de végétation en Auvergne.

b) Rameaux entiers (fig. 14 à 17).

Leur comportement est différent de celui des boutures.

$1^{\circ}$ Dans tous les cas le nombre de bourgeons développés reste faible. On retrouve mieux caractérisée encore cette particularité déjà signalée chez le Tilleul.

$2^{\circ}$ En octobre il y a deux centres de préséances, apical et basal, séparés par une série de points végétatifs plus dormants.

$3^{\circ}$ Ce gradient bipolaire est remplacé en janvier par une tendance à l'acrotonie qui se maintient jusqu'en mars à presque toutes les températures, et qui rend donc compte, mieux que chez le Tilleul du comportement dans la nature.

On peut remarquer enfin que le nombre de débourrements en février est inférieur à celui d'octobre: il s'agit d'un fait mal expliqué qui retient notre attention ; tout se passe comme si l'intensité de la dormance, malgré les premiers froids, augmentait au début de l'hiver pour se retrouver, fin février, au niveau qui était le sien en automne.

c) Comportement des jeunes plantes (fig. 18).

$1^{\circ}$ Sur les boutures de nœuds isolés prélevées sur des jeunes plantes de 3 à 6 ans et bien que ces dernières soient constituées de segments d'âge différent, la basitonie est nette en automne et fait place à la fin de l'hiver à une égalisation des chances de développement. Le gradient basitone persiste donc un peu moins que sur les rameaux vigoureux. L'analogie avec le Tilleul est totale.

En l'absence de système radiculaire aucun débourrement ne se produit pendant la durée de l'expérience. Les causes de cette augmentation anormale du temps de latence nous échappent encore et sont à l'étude.

$2^{\circ}$ Sur les plantes entières, en présence du système radiculaire, la reprise de croissance est rapide et le renversement des préséances très net: on observe avant la fin de l'hiver une intense acrotonie: seul le bourgeon terminal redevient actif. Il est certes de beaucoup le plus gros. Cette préséance morphologique ne s'exprime cependant pas sur les nœuds isolés.

Ce résultat doit être considéré comme normal si on se souvient que, dans la nature, sur les plantules, le bourgeon terminal entre seul en croissance et poursuit l'édification du tronc (acrotonie de débourrement). Son intérêt est de montrer clairement que l'intégrité de l'axe, même s'il est constitué de segments annuels superposés, est indispensable pour que cet important caractère se manifeste. C'est une autre preuve de l'importance des corrélations hivernales entre bourgeons partiellement dormants pour l'expression des tendances morphogènes des végétaux ligneux acrotones. 


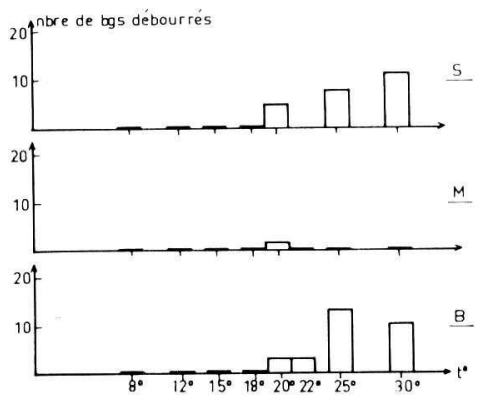

Fig. 14

Rameaux entiers de FRÊNE FÉVRIER

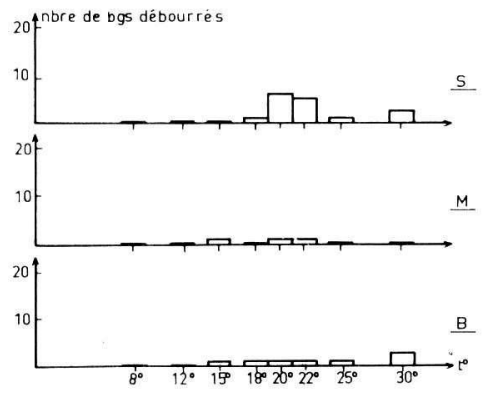

FIG. 16

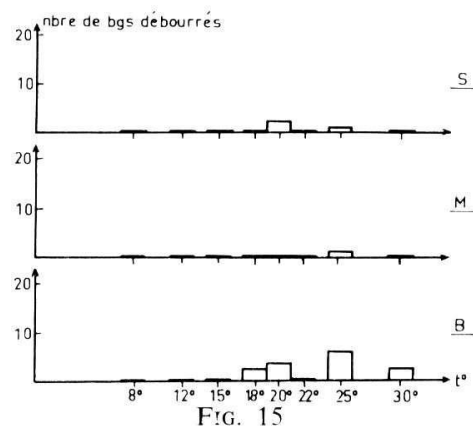

Rameoux entiers de FRENNE MARS

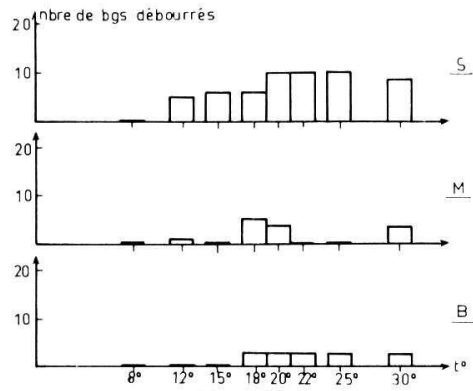

FIG. 17

FIG. 14 à 17. - Débourrement des bourgeons sur des rameaux entiers de Fraxinus excelsior prélevés dans la nature entre octobre et mars et maintenus à différentes températures (abscisses)

Chaque série comprend 20 rameaux (ordonnées). Les résultats sont réevés 40 à 50 jours après la mise en expérience. Ils découlent de l'observation de 3 paires de bourgeons de chaque pousse: une paire apicale $(\mathrm{S})$, une paire médiane $(\mathrm{M})$ et une paire basale $(\mathrm{B})$, ce qui facilite la comparaison avec les boutures de nœuds isolés (fig. 10 à 13).

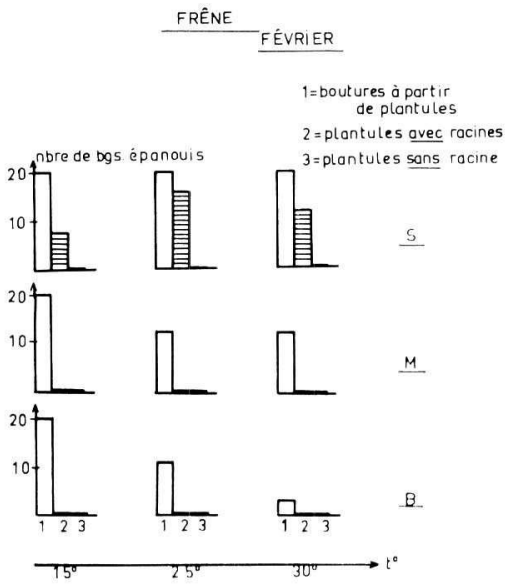

FIG. 18

Fig. 18. - Comportement comparé de boutures de nouds isolés (1), prélevés sur de jeunes plantes en février, et de témoins entiers conservant (2) ou non (3) leur système radiculaire. Les coordonnées et la durée de l'expérience sont les mêmes que dans les figures précédentes 


\section{C. - Cas du Cerisier}

a) Boutures de nouds isolés (fig. 19 à 21).

L'évolution de leur comportement d'octobre à mars est analogue à celle des autres espèces: à une médio-basitonie très nette en octobre et qui se maintient, en s'atténuant un peu, jusqu'en janvier, fait suite, dès février, une parfaite égalisation des chances de développement de tous les bourgeons sauf à $8^{\circ} \mathrm{ou}$, comme chez le Tilleul, les méristèmes apicaux ne reprennent pas leur croissance et à $30^{\circ}$ où tous restent latents.

Ces résultats ne rendent pas compte de l'acrotonie caractérisée qu'on observe dans la nature.

b) Rameaux entiers (fig. 22 et 23).

On retrouve la plupart des faits déjà signalés; faible proportion de bourgeons capables de se développer pendant la plus grande partie de l'automne et de l'hiver ; tendance basitone en octobre entre $20^{\circ}$ et $25^{\circ}$, disparition de cette tendance en janvier.

Toutefois, en février, ce n'est pas l'acrotonie attendue qui s'exprime, mais un gradient à deux maximums peu marqués, les bourgeons médians se développant avec un léger retard par rapport à ceux des deux extrémités. Ce gradient s'efface d'ailleurs en mars comme chez le Tilleul. De nouvelles recherches sont donc nécessaires pour préciser si, dans la nature, l'origine de la préséance apicale est dans le système radiculaire ou dans certaines branches plus âgées. Par analogie avec des résultats inédits obtenus sur le Rhamnus et avec le cas des jeunes frênes, la première possibilité est la plus probable.

\section{D. - Cas du Charme}

a) Boutures de nouds isolés (fig. 24 et 25).

Le seul fait à signaler est le maintien de la méso-basitonie pendant toute la durée des expériences. En mars elle est encore remarquablement nette. Même chez un Rubus elle a, à ce moment, disparu. Il reste, cependant, difficile de la mettre en relation avec les tendances basitones de l'espèce. La basitonie ne s'exprime, en effet, dans la nature, par aucun gonflement automnal ou hivernal des bourgeons de base, certains d'entre eux, au contraire resteront latents lors du débourrement.

b) Rameaux entiers (fig. 26 à 28).

Ils rendent compte comme chez le Tilleul et le Frêne de la ramification naturelle. En octobre peu de bourgeons débourrent selon un faible gradient basitone à $20^{\circ}$ et $22^{\circ}$, qui tend d'ailleurs à s'inverser à $30^{\circ}$. En janvier les chances de développement de chaque point végétatif sont les mêmes tout le long de l'axe malgré le grand nombre de ceux qui restent en repos pendant toute la durée de l'expérience. En mars, enfin, la croissance est rapide et générale áans les 1/3 moyen et supérieur alors que l'inhibition est forte à la base. 


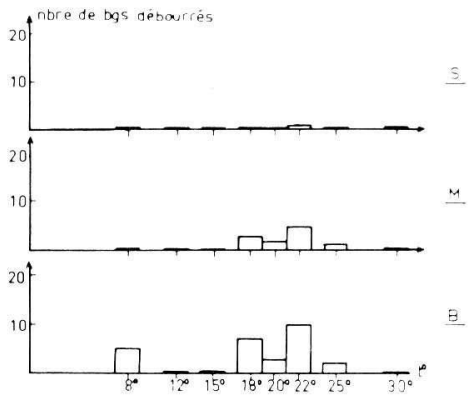

FIG. 19

Boutures de CERISIER FEVRIER

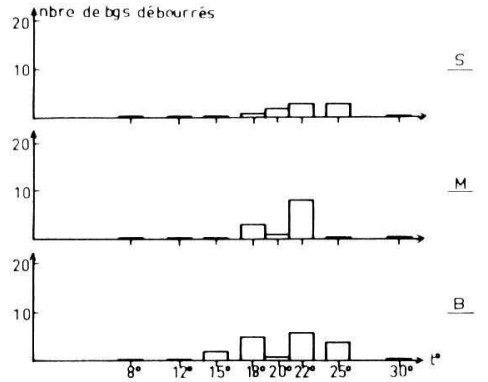

FIG. 20

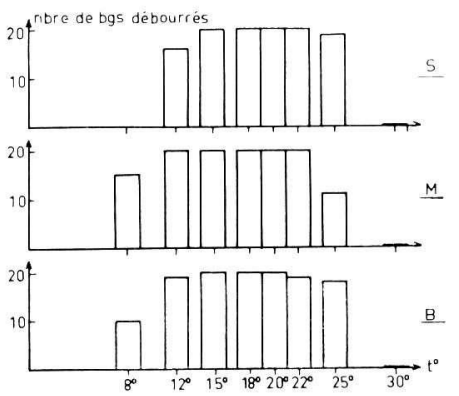

FIG. 21

FIG. 19 à 21. - Débourrement des bourgeons sur des boutures de næuds isolés de Cerasus avium, prélevées dans la nature entre octobre et mars et maintenues à différentes températures (abscisses)

Chaque série comprend 20 boutures (ordonnées). Les résultats sont relevés 40 à 50 jours après la mise en expérience.

S; M ; B: boutures prélevées respectivement dans les régions apicale, médiane et basale des rameaux.

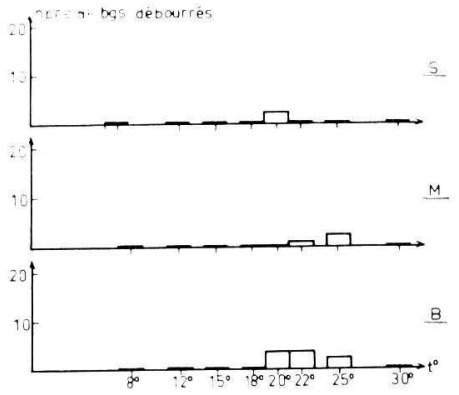

FIG. 22

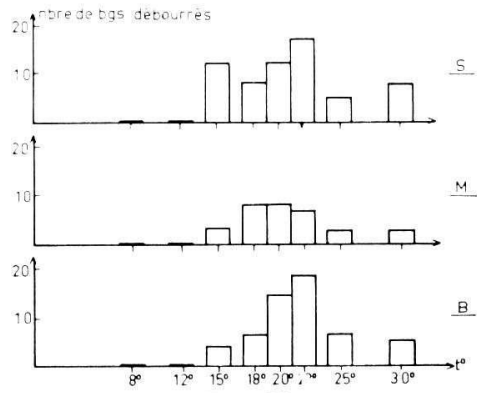

FIG. 23

FIG. 22 et 23. - Débourrement des bourgeons sur des rameaux entiers de Cerasus avium prélevés dans la nature entre octobre et mars et maintenus à différentes températures (abscisses).

Chaque série comprend 20 rameaux (ordonnées). Les résultats sont relevés 40 à 50 jours après la mise en expérience. Ils découlent de l'observation de 3 paires de bourgeons de chaque pousse : une paire apicale $(\mathrm{S})$, une paire médiane $(\mathrm{M})$ et une paire basale $(\mathrm{B})$, ce qui facilite la comparaison avec les boutures de nœuds isolés (fig. 19 à 21). 


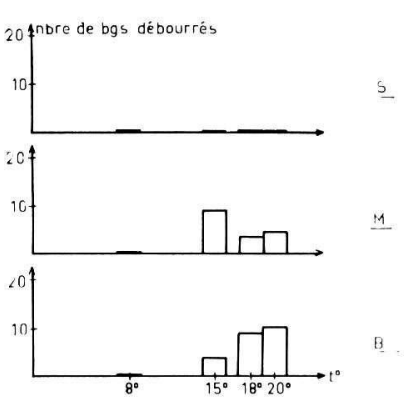

FIG. 24

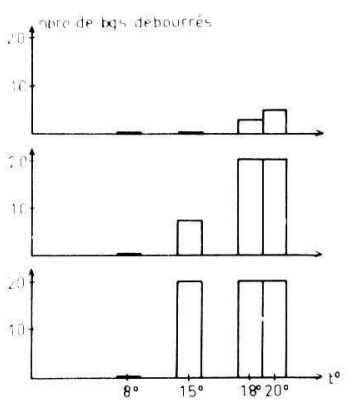

FIG. 25

FIG. 24 et 25. - Débourrement des bourgeons sur des boutures de roeuds isolés de Carpinus betulus, prélevées dans la nature entre octobre et mars et maintenues à différentes températures (abscisses).

Chaque série comprend 20 boutures (ordonnées). Les résultats sont relevés 40 à 50 jours après la mise en expérience.

$\mathrm{S} ; \mathrm{M}$; B; boutures prélevées respectivement dans les régions apicale, médiane et basale des rameaux.

Rameouxentiers de CHARME

OCTOBRE

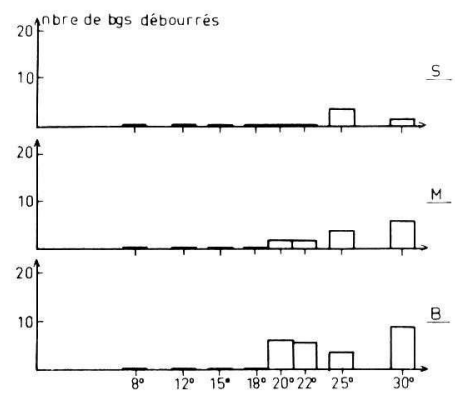

FIG. 26
Rameoux entiers de CHARME JANVIER

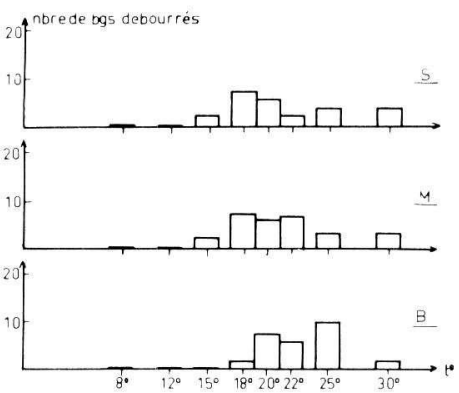

FIG. 27

Romeaux entiers de CHARME MARS

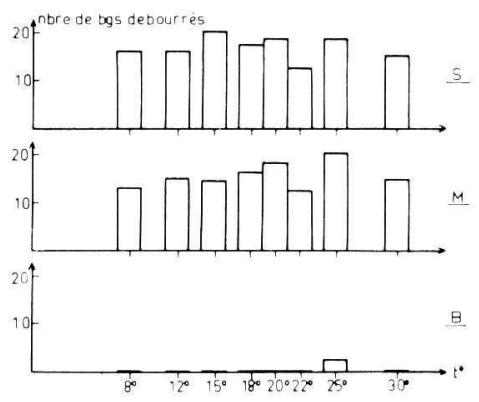

FIG. 28

FIG. 26 à 28. - Débourrement des bourgeons sur des rameaux entiers de Carpinus beiulus, prélevés dans la nature entre octobre et mars et maintenus à différentes températures (abscisses)

Chaque série comprend 20 rameaux (ordonnées). Les résultats sont relevés 40 à 50 jours après la mise en expérience. I's découlent de l'observation de 3 paires de bourgeons de chaque pousse: une paire apicale $(S)$, une paire médiane $(M)$ et une paire basale $(B)$, ce qui facilite la comparaison avec les boutures de nouds isolés (fig. 24 et 25). 


\section{CONCLUSIONS ET PERSPECTIVES}

$1^{\circ}$ Le long d'un rameau, pendant toute la durée de la mauvaise saison, sur toutes les espèces étudiées, on met en évidence des différences dans l'aptitude des divers bourgeons isolés à répondre par une croissance à des conditions favorables. Ce gradient d'aptitude n'est pas stable: à une basitonie automnale très générale succède un développement plus homogène, plus rarement suivi par une tendance acrotone qui n'est jamais aussi bien caractérisée que celle observée par CrabBé sur le Pommier et le Poirier et qui ne peut pas rendre compte de la ramification normale des pousses au printemps.

$2^{\circ}$ La basitonie automnale est identique à celle mise en évidence par BARNOLA sur divers buissons. Mais alors que dans ce cas elle se traduit rapidement par une évolution morphologique génératrice de préséances définitives, base de l'édification d'une touffe, elle est ici sans conséquence. Un autre facteur morphogène, plus puissant qu'elle, l'empêche de s'exprimer. C'est l'existence de ce deuxième facteur, qui, pour l'instant, différencie le mieux arbres et buissons. Il est vraisemblable que si on pouvait diminuer son importance on modifierait profondément le port d'espèces habituellement aptes à différencier un tronc.

$3^{\circ}$ Sur un rameau entier, si la basitonie est décelable en octobre, et si une absence de préséances lui succède, on observe souvent, en mars, une acrotonie suffisante pour rendre compte de la morphologie observée dans la nature lors du débourrement (acrotonie par croissance différentielle du Tilleul, des rameaux vigoureux du Frêne et du Charme, et même acrotonie stricte des plantules de Frêne). L'intégrité de la pousse, donc certains rapports mutuels entre les bourgeons, renforce ainsi l'acrotonie et est fondamentale pour l'expression du deuxième facteur de préséances mentionné au paragraphe précédent.

$4^{\circ}$ On peut penser que ce facteur «rameau entier» est puissant chez la Vigne dès le début de l'automne (Huglin, 1958) et existe aussi chez le Framboisier (BARNola, 1969). Mais, dans ces deux cas, à l'encontre des espèces étudiées ici, il disparaît au cours de l'hiver au lieu de se renforcer. Cette particularité a besoin d'être expliquée et retiendra désormais notre attention.

$5^{\circ}$ Dans certains cas, ce facteur ne suffit pas pour rendre compte de l'intensité de l'acrotonie [Cerisier, Chêne (LAVARenne-Allary, 1965)]. Il est vraisemblable que les corrélations avec des organes plus différenciés (système radiculaire, grosses branches) sont alors seules capables de le renforcer suffisamment.

$6^{\circ}$ L'acrotonie n'est de toutes façons pas un phénomène comparable à la basitonie. Elle ne s'installe jamais directement dès l'entrée en dormance des bourgeons. Elle est secondaire et succède à une préséance des yeux inférieurs. Elle met en jeu (d'une manière variable suivant les espèces) aussi bien les propriétés intrinsèques de chaque bourgeon, que celles du rameau entier ou même, comme nous venons de le mentionner, des corrélations entre organes de nature différente.

$7^{\circ}$ Bien des faits, outre ceux qui viennent d'être signalés, restent inexpliqués: causes du faible pourcentage de débourrement sur les rameaux entiers pendant la 
première partie de l'hiver, renforcement apparent de la dormance en novembre, action parfois très différente de températures voisines.

$8^{\circ}$ Notre but essentiel reste cependant de rechercher la nature des divers gradients mis en évidence, de ces facteurs de préséances qui contrôlent les caractères généraux de la morphogenèse des végétaux ligneux. Leur pluralité et leur coopération durant les mois où la vie, sous nos climats, est ralentie, étaient inattendues. Leur nature semble, à première vue, difficile à expliquer à l'aide de mécanismes connus.

$9^{\circ}$ Nous ne perdrons pas de vue, que sous des climats tropicaux et équatoriaux, les arbres et les buissons s'édifient selon des principes stricts qui supposent parfois d'intenses préséances entre bourgeons (Attims, 1969 ; Halle et Oldeman, 1970 ; Prévost, 1966; Scarrone, 1969). L'existence en ces lieux comme en chambres climatisées, d'une croissance rythmique [Lavarenne-Allary, 1966; Lavarenne et al. (sous presse)], fréquente malgré des conditions uniformes de milieu, donne à penser qu'une variation cyclique des potentialités de croissance est un caractère très général des méristèmes. Il sera intéressant de savoir si elle intéresse de façon synchrone tous ceux que porte une même tige et si on peut, dans ce cas, parler d'une dormance bien distincte d'un dominance apicale.

$10^{\circ}$ Nous ne perdrons pas de vue, non plus, que des gradients longitudinaux, de préséances se traduisent parfois par des différenciations plus poussées qu'une aptitude à la croissance: un plagiotropisme plus ou moins irréversible, une morphologie diversifiée des organes produits par les méristèmes. Divers exemples de cette sorte ont été remarquablement étudiés par Nozeran et ses collaborateurs (BANCILlon, 1969 ; Neville, $1969 a$ et $b$; Roux, 1968). A notre avis, ces spécialisations pourraient s'ajouter à celles, plus simples et très répandues que nous étudions. Elles sont toutefois beaucoup plus intimement liées à une dominance apicale typique, à des corrélations actives entre organes très jeunes, en un mot, à la physiologie de la pousse herbacée en croissance. Elles ne s'en traduisent pas moins, l'année suivante, par une détermination pratiquement irréversible de certaines potentialités: rameaux longs, donc apparemment dominants, (orthotropes plagiotropes, rameaux courts très spécialisés) (HaLle et OldEman, 1970 ; Roux, 1968 ; Thimann et al., 1949 ; Titman et Wetmore, 1955) qui ne sauraient être essentiellement différentes des acrotonies ou basitonies plus labiles qui nous préoccupent.

Reçu pour publication en janvier 1971.

\section{SUMMARY}

PRELIMINARY RESEARCHES ON THE FACTORS

DETERMINING ACROTONY IN WOODY PLANTS

Acrotony, wrongly named «apical dominance» by many authors, is the essential principle of the building up of the trunk and of the main branches of trees. On the one year shoot only the apical buds produce long ramifications, the others giving short twigs or remaining dormant. Acrotony is in opposition to basitony which conditions the bearing of bushes.

Generally the precedence of apical buds does not exist on a leafy shoot when it completes its growth and enters into tree dormancy.

On the contrary, at the beginning of autumn, cuttings without roots of isolated nodes show that the buds at the base are most able to grow: their winter dormancy is the less deep 
and this is shown by a faster, more homogeneous opening of buds on a larger scale of temperatures, notably at about $12^{\circ}$ to $15^{\circ} \mathrm{C}$. During that season, the isolated nodes of the trees (Fraxinus excclsior, Tilia platyphyllos, Prunus avium, Carpinus betulus) and the bushes (Rubus, Sambucus nigra, Rhamnus frangula) have ine same behavior.

From december to march, on the same cuttings the aptitude of the buds to grow, increases and become the same whatever has been their place on the seem. So, on th? trees the basitone gradient shown in autumn disappears while it remains on some bushes after being set in a concrete form by an important growth of some buds at the base.

That comparable growth ability of numerous buds on the shoot, at the end of winter, allow us to understand the cases of acrotony «by differential growth » in which the development of young sprouts stays uniform for a rather long time, before those at the top acquire, progressively, their precedence.

It does not take account of the «bursting buds» acrotony in which that precedence reveals itself from the beginning of the opening of the buds. If in a few cases (apple trees studied by CRABBÉ) a gradient of acrotony is set in february-march and seen on the one node cuttings, in the greatest number of cases it is a factor completely different, which conditions the expression of acrotony: the integrity of the one year shoot. When cuttings of isolated buds have an identical behaviour or if even, they exhibit basitony, the whole shoots, set in favourable conditions for growth, reveal themselves as acrotones. Sometimes the presence of the radicular system strengthens this tendency.

Thus, mutual relations between buds, adequate correlative influences are in action during the course of winter, when no growth is visible, and they are of a great importance in the morphogenesis of woody plants.

Therefore acrotony is not a phenomenon directly comparable with basitony. It results from an evolution of the specific properties of each bud as well as from that of the entire shoot.

The essential problem remains to understand the nature of these gradients and of the correlations active during winter, nature difficult to explain by means of known mechanisms.

\section{ZUSAMMENFASSUNG}

\section{ERSTE UNTERSUCHUNGEN ÜBER DEN DETERMINISMUS}

\section{DER AKROTONIE BEI HOLZGEWÄCHSEN}

Im Klima Mittelfrankreichs ist die Dominanz der apikalen Knospen, welche die Akrotonie der 4 untersuchten Baumarten bestimmen, nicht vor dem Monat März gegeben. Sie bedingt aktive Wechselwirkungen zu einem Zeitpunkt, an dem noch kein Wachstum festzustellen ist: der Vergleich der Entwicklung bei Stecklingen mit einzelnen Knoten und bei ganzen Zweigen liefert den Beweis, dass nach einer Herbstbasitonie, die jener der Sträucher gleich ist (Rubus, Sambucus, unsw...), eine ebenwertige Entwicklungsfähigkeit aller Knospen folgt und dann eine apikale Dominanz, die weit weniger ausgeprägt ist bei den einzelnen Knoten als bei dem ganzen Zweige. Die Unversehrtheit des Zweiges ist also ein wichtiger Faktor der Akrotonie, die das Wurzelsystem auch noch verstärken kann.

Die Akrotonie ist also nicht direkt mit der Basitonie vergleichbar: sie folgt ihr und ersetzt sie. Sie erbringt neue Faktoren, deren Natur noch nicht ergründet ist, und die eben die basitonale Tendenz daran hindern, sich zu entfalten.

Diese Ergebnisse bestätigen ferner das ständige Vorhandensein von verschieden starken Hemmungsfaktoren längs dem Zweige und eine von Oktober bis März fortschreitende Veränderung des so entstandenen Wachstumsgradienten.

\section{RÉFÉRENCES BIBLIOGRAPHIQUES}

Aтtims Y., 1969. Morphogenèse de quelques espèces du Genre Gossypium L. Rapport O.R.S.T.O.M., Abidjan, 29 pages.

Barnola P., 1969. Recherches sur le déterminisme de la basitonie dans le genre Rubus, Thèse $3^{\mathrm{e}}$ cycle, Clermont-Ferrand, $157 \mathrm{p}$.

Barnola P., 1970. Recherches sur le déterminisme de la basitonie chez le Framboisier (Rubus idaeus L.). Ann. Sci. nat. Botanique, Paris, $12^{e}$ série, XI, p. 129-151.

Barnola P., 1971. Recherches sur le déterminisme de la basitonie chez Sambucus nigra (L.) observé en conditions contrôlées, (à paraître). 
Bancilhon L., 1969. Etude expérimentale de la morphogénèse et plus spécialement de la floraison d'un groupe de Phyllanthus (Euphorbiacées) à rameaux dimorphes. Ann. Sci. nat. Botanique, Paris, $12^{\mathrm{e}}$ série, $\mathbf{X}$, p. 127-224.

BEN AMOR T., 1970. Recherches sur la dormance des bourgeons de la vigne en Tunisie. Thèse, $3^{\text {e }}$ cycle, Clermont-Ferrand, $166 \mathrm{p}$.

Bugnon F. \& R. BEssis, 1968. Biologie de la Vigne. Acquisitions récentes et problèmes actuels, 1 vol., Masson \& $\mathrm{C}^{\mathrm{ie}}$, Paris, $160 \mathrm{p}$.

Champagnat P., 1954. Les corrélations sur le rameau d'un an des végétaux ligneux. Phyton (Argentine), 4, p. 1-102.

Champagnat P., 1965. Physiologie de la croissance et de l'inhibition des bourgeons: dominance apicale et phénomènes analogues. Encyclopedia of Plant Physiology, 15 (1), p. 1106-1164.

Champagnat P., 1965. Quelques caractères de la ramification du rameau d'un an des végétaux ligneux. C.R., 96 Congrès Soc. pomol. France, 9-33.

Champagnat P., 1969. La notion de facteurs de préséances entre bourgeons. Bull. Soc. bot. Fr., 116, p. 323-348.

Champagnat P., Allary S. et Randier A.-M., 1961. La ramification de la pousse herbacée du Charme et le problème des rameaux anticipés et des pousses de la Saint-Jean. Mem. Soc. Bot. Fr., p. 81-91.

Crabbe J., 1968. Evolution annuelle de la capacité intrinsèque de débourrement des bourgeons successifs de la pousse de l'année chez le Pommier et le Poirier. Bull. Soc. roy. bot. Belgique, 101, p. 195-204.

Halle F. \& Oldeman R.-A., 1970. Essai sur l'Architecture et la dynamique de la croissance des arbres tropicaux, 1 vol., Masson \& $C^{\text {ie }}$, Paris, 178 p. Huglin P., 1958. Recherches sur les bourgeons de la Vigne: initiation florale et développement
végétatif. Thèse, Strasbourg, $174 \mathrm{p}$.

Lavarenne-Allary S., 1965. Recherche sur la croissance des bourgeons du chêne et de quelques autres espèces ligneuses. Ann. Sci. Forest., 22, 1-203, (Nancy).

Lavarenne-Allary S., 1966. Croissance rythmique de quelques espèces de Chênes cultivés en chambres climatisées. C.R. Acad. Sc., t. 262, 358-361.

Lavarenne S., Champagnat P., Barnola P. Croissance rythmique de quelques végétaux ligneux des régions tempérées cultivés en chambres climatisées à température élevée et constante Bull. soc. bot. France (sous presse).

Neville P., 1969. Morphogénèse chez Gleditsia triacanthos. I. Mise en évidence expérimentale de corrélations jouant un rôle dans la morphogénèse et la croissance des bourgeons et des tiges. Ann. Sci. nat. Bot., $12^{\mathrm{e}}$ série, IX, p. 433-510.

Neville P., 1969. Morphogénèse chez Gleditsia triacanthos. II. Etude expérimentale de la spinescence. Ann. Sci. nat. Bot., $12^{\mathrm{e}}$ série, X, p. 103-126.

Nozeran R., Bancillon L. \& Neville P. Intervention de corrélations internes dans la morphogénèse des végétaux supérieurs. Advances in Morphogenesis (sous presse).

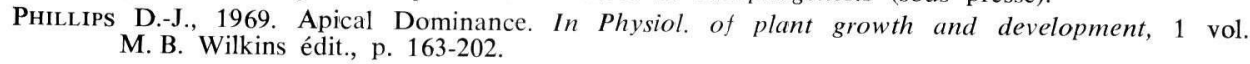

Prévost M.-F., 1966. Etude de la morphogénèse de quelques buissons de Côte d'Ivoire. Rapport O.R.S.T.O.M., Abidjan, 22 p. Prévost M.-F., 1966. Architecture de quelques Apocynacées ligneuses. Mém. Soc. bot. Fr., 114,
p. 23-26.

RaUH W., 1939. Über Gesetzmässigkeit der Verzweigung und deren Bedeutung für die Wuchsformen der Pflanzen. Mitt. Dtsch. Deudr. Ges., 52, 86-111.

Koux J., 1968. Sur le comportement des axes aériens chez quelques plantes à rameaux végétatifs polymorphes. Le concept de rameau plagiotrope. Ann. Sci. nat. Bot., Paris, $12^{\mathrm{e}}$ série, $\mathbf{9}$,
p. 227-284.

SCARrone F., 1969. Recherches sur les rythmes de croissance du Manguier et de quelques végétaux ligneux malagasy, Thèse, Clermont-Ferrand, 2 vol., 438 et 248 p.

Thimann K. V., Gunckel J. E. and Wetmore R. H., 1949. Studies of development in long shoots and short shoots of Ginkgo biloba. IV. Growth habit, shoot expression and the mechanism of its control. Amer. J. Bot., 36, p. 309-318. Troll W. Vergleichende Morphologie der höheren Pflanzen. Bd. 1, Teil 1, Berlin 1937 et Bd. 1,
Teil 2, Berlin 1939.

Vegis A., 1961. Samenkeimung und Vegetative Entwicklung der Knospen. Encyclopedia of Plant Physiology, 16, 168-298.

Vegis A., 1965. Die Bedeutung von physikalischen und chemischen Aussenfaktoren bei der Induktion und Beerdigung von Ruhezuständen bei Organen und Geweben höherer Pflanzen. Encyclopedia of Plant Physiology, 15, (2), 534-668. Titman P. W. and Wetmore R. H., 1955. The growth of long and short shoots in Cercidophyllum.
Amer. J. Bot., 42, 364-372. 